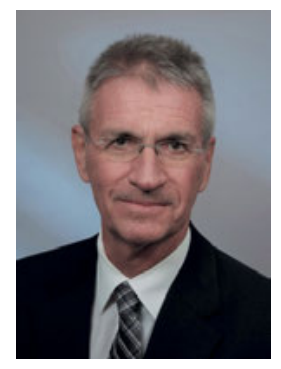

\title{
Vorwort zur 4. Auflage
}

Die 4. Auflage „Steuern“ beinhaltet eine vollständige Überarbeitung dieses insbesondere an Studierende von Bachelor-Studiengängen gerichteten Werkes. Mit der Neugestaltung der „Betriebswirtschaftlichen Steuerlehre“, die vor allem Studierende von Master-Studiengängen anspricht und die in 8. Auflage (erschienen 2020 mit Rechtsstand 2020) im gleichen Verlag verfügbar ist, besteht damit eine Einheit, die den gesamten Stoffumfang des Faches „Betriebswirtschaftliche Steuerlehre" abbildet. Das Buch befindet sich auf dem gesetzlichen Stand des Jahres 2020, wobei alle Änderungen, welche bis zum 01.07.2020 vorgenommen worden sind, berücksichtigt wurden.

Das Werk wendet sich an Leser, die sich grundlegend und umfassend mit Fragen der Steuerarten inklusive der rechtsformorientierten Besteuerung auseinandersetzen wollen, seien es Lehrende und Studierende an Universitäten, Fachhochschulen, Dualen Hochschulen, Verwaltungs- und Wirtschaftsakademien und anderen Einrichtungen, seien es interessierte Praktiker. Der Stand des Faches wird in Kombination der beiden Bücher in ausführlicher Weise wiedergegeben; dabei wird auf der einen Seite eine - insgesamt in möglichst knapper Form erfolgende - Darstellung des Stoffes angestrebt, auf der anderen Seite wird der Bezug zu einem konkreten Steuerrechtsstand hergestellt, sodass auch Detailbetrachtungen erfolgen können.

Für die umfangreichen Vorarbeiten in Form zahlreicher Hinweise, Hilfen und Verbesserungsvorschläge gilt mein besonderer Dank Herrn Dr. DANIEL LiCHT (Gliederungspunkte 1 bis 4 sowie 8 bis 10), Herrn StB Dr. Florian MÜLleR (Gliederungspunkte 5 und 6) sowie Frau StB Dr. Chantal Naumann (Gliederungspunkte 7 und 11). Für die von Zielstrebigkeit und Kompetenz getragene Unterstützung bei der konkreten Fertigstellung dieses Projekts im Kontext von Koordinierungs- und Formalisierungsaufgaben inklusive der Erstellung einer Druckvorlage für den Verlag gilt mein großer Dank Herrn Dr. DANIEL LiCHT sowie für die abschließenden inhaltlichen und redaktionellen Arbeiten Frau StB Dr. Chantal NaumanN, welche diese Aufgaben mit unermüdlichem Einsatz und sehr großer Kompetenz erfüllt haben. Für die Mithilfe im gesamten Umfeld der Publikation geht mein ganz besonderes Dankeschön 
an Frau HeIKE MANG, für die harmonische Zusammenarbeit mit dem Verlag bedanke ich mich bei Herrn Dr. Stefan Giesen, Frau Angelika Hermann und Frau Lucy Jarman.

Saarbrücken, im Juli 2020

HEINZ KUßMAUL 